\title{
Recent NA61/SHINE measurements performed for the T2K experiment
}

\author{
Magdalena Posiadała-Zezula for the NA61/SHINE Collaboration ${ }^{1, a}$ \\ ${ }^{1}$ University of Warsaw, Faculty of Physics, Pasteura 5, $02-093$ Warsaw, Poland
}

\begin{abstract}
The neutrino programme of the NA61/ SHINE experiment at the CERN SPS is aiming to deliver precise hadron production measurements for improving calculations of the initial neutrino beam flux in the long-baseline neutrino oscillation experiments. The first receiver of such measurements is the T2K neutrino oscillation project in Japan. New results on $\pi^{ \pm}, K^{ \pm}, \mathrm{p}, K_{S}^{0}$ and $\Lambda$ production from the NA61/SHINE 2009 thin target data analyses with smaller statistical and systematic errors are discussed. They enable us to reduce further the flux uncertainties in $\mathrm{T} 2 \mathrm{~K}$ for neutrino and antineutrino beam mode. We also report on the first corrected $\pi^{ \pm}$results obtained for T2K replica target (a $90 \mathrm{~cm}$ long cylinder of $2.6 \mathrm{~cm}$ diameter, about $1.9 \lambda_{I}$ ). Up to $90 \%$ of the neutrino flux can be constrained by such measurements as compared to $60 \%$ for the thin target measurements that are sensitive only to primary hadron interactions.
\end{abstract}

\section{Introduction}

The NA61/SHINE (SHINE = SPS Heavy Ion and Neutrino Experiment) combines a rich physics program in three different fields: auxiliary measurements for neutrino experiments, cosmic-ray simulations, and the behaviour of strongly interacting matter at high density. In its first stage of data taking (years 2007, 2009 and 2010) NA61/SHINE performed measurements of $\mathrm{p}+\mathrm{C}$ interactions at $31 \mathrm{GeV} / \mathrm{c}$ needed for neutrino experiment Tokai-2-Kamioka (T2K).

\section{The T2K experiment}

The T2K is a long baseline neutrino oscillation experiment which uses an intense proton beam produced by the J-PARC (Japan Proton Accelerator Research Complex) in Tokai, Japan. It is composed of neutrino beamline, near detector complex, both of which were newly constructed at J-PARC and far detector Super-Kamiokande, located $295 \mathrm{~km}$ away in Mozumi mine. Comprehensive review of the instrumentation aspects of the T2K experiment may be found in Ref. [1]. The T2K can measure neutrino oscillation parameters in two channels. The first is muon neutrino disappearance, which provides measurements of the parameters: $\theta_{23}$ and the largest mass neutrino splitting. The second is electron neutrino appearance, which provides measurements of the parameters: $\theta_{13}$ and, also, the CP-violating phase $\delta_{C P}$.

ae-mail: Magdalena.Posiadala@fuw.edu.pl 


\subsection{The T2K beam production}

The J-PARC neutrino beamline has two complementary subsystems. These are: the primary and secondary beamlines. The main aim of the primary beamline is to tune proton beam coming from J-PARC accelerator, bent it towards the direction of Kamioka, and focus the beam onto the target. In the secondary beamline we can distinguish three sections: the target station, decay volume and beam dump. The target station is equipped with three magnetic horns and the graphite target, 90 $\mathrm{cm}$ in length, which is inserted inside the first one. When proton beam is impinging on the graphite target many hadrons are produced, amongst of which charged pions and kaons are crucial as neutrino parents. At the beginning of the T2K data taking horns were working at $250 \mathrm{kA}$ current pulse in order to focus positively charged hadrons to produce a muon neutrino enhanced beam $v_{\mu}$. An anti-neutrino enhanced beam $\bar{v}_{\mu}$ is produced by T2K starting from 2013. Experimentally it is achieved by reversing the current direction in the focusing horns in order to select negatively charged particles. Precision of the T2K beam simulation relies heavily on the knowledge of the hadron production of neutrino parents produced in $\mathrm{p}+\mathrm{C}$ interactions at $31 \mathrm{GeV} / \mathrm{c}$. This is the reason why neutrino programme of the NA61/SHINE experiment is essential for T2K.

\section{The NA61/SHINE experiment}

The NA61/SHINE apparatus is a wide-acceptance hadron spectrometer at the CERN SPS. The detector is built around five Time Projection Chambers (TPCs). Two Vertex TPCs are placed in the magnetic field produced by two superconducting dipole magnets and two Main-TPCs are located downstream symmetrically with respect to the beamline. An additional small TPC is placed between Vertex TPCs, covering the very forward region, and is referred to as the GAP TPC (GTPC). The GTPC is used for tracking in the analysis described here allowing to extend the kinematic coverage compared to our previously published results. Since 2007 pilot run the experiment has been updated with a new forward time-of-flight detector (ToF-F) in order to extend the acceptance for pion and kaon identification as required for T2K measurements [2,3]. The NA61/SHINE experimental facility is described in detail in Ref. [5]

Data needed for the T2K experiment were collected using two graphite targets:

- the $2 \mathrm{~cm}$ long (about 0.04 of interaction length $\lambda_{I}$ ) so called thin target,

- the T2K replica target (a $90 \mathrm{~cm}$ long cylinder of $2.6 \mathrm{~cm}$ diameter, about $1.9 \lambda_{I}$ ).

A summary of NA61/SHINE data collected for T2K is presented in Table 1. Results of $\pi^{ \pm}$and $K^{+}$yields from proton-carbon interactions at $31 \mathrm{GeV} / \mathrm{c}$ taken in 2007 pilot run with a thin graphite target were already used by the T2K experiment to tune neutrino beam simulations and reduce uncertainties( [6-11]). In this article we want to emphasise recently published results from the thin target data set obtained from 2009 run [12]. Moreover, T2K replica target results, from 2009 dataset, which were also recently accepted for publication [14] are discussed.

\subsection{The NA61/SHINE 2009 thin target results}

New thin target results from the 2009 data set on $\pi^{ \pm}, K^{ \pm}, \mathrm{p}, K_{S}^{0}$ and $\Lambda$ were obtained in bins of momentum $p$ and production angle $\theta$. In Fig. 1 we present the kinematic region of interest of neutrino parents for the T2K. Four panels represent the momentum $p$ and $\theta$ distributions of $\pi^{ \pm}$and $K^{ \pm}$whose daughter neutrinos pass through the Super-Kamiokande detector. These studies apply to neutrino beam mode configuration in T2K. On all figures the NA61/SHINE coverage from 2007 pilot run (dashed line) and 


\begin{tabular}{|c|c|c|c|c|}
\hline target & year & $\begin{array}{c}\text { triggers } \\
\times 10^{6}\end{array}$ & $\begin{array}{c}\text { status of the } \\
\text { NA61/SHINE analysis }\end{array}$ & $\begin{array}{c}\text { usage in } \\
\text { the T2K beam MC }\end{array}$ \\
\hline \hline thin target & 2007 & 0.7 & published: $\pi^{ \pm}[2], K^{+}[3], K_{S}^{0}, \Lambda[4]$ & has already been used [11] \\
\cline { 3 - 5 } $2 \mathrm{~cm}\left(0.04 \lambda_{\mathrm{I}}\right)$ & $\mathbf{2 0 0 9}$ & 5.4 & $\pi^{ \pm}, K^{ \pm}, \mathrm{p}, K_{S}^{0}, \Lambda[12]$ & currently being used \\
\hline T2K replica & 2007 & 0.2 & published: $\pi^{ \pm}[13]$ & method developed \\
target & 2009 & 2.8 & $\pi^{ \pm}$analysis finalised [14] & to be integrated \\
\cline { 3 - 5 } $90 \mathrm{~cm}\left(1.9 \lambda_{\mathrm{I}}\right)$ & 2010 & 7.2 & analysis currently on-going & - \\
\hline
\end{tabular}

Table 1: A summary of NA61/SHINE data collected for T2K physics goals.

2009 run (solid line) is also included. One may conclude that the improved statistics of the 2009 data (by a factor of 8 larger with respect to 2007 data set) allows for the first measurements of negatively charged kaons within NA61/SHINE. Overall precision of the measurements is also improved. Moreover, the acceptance of the pions is also extended in comparison to 2007 data set. Similar results for T2K anti-neutrino beam mode may be found in [12].

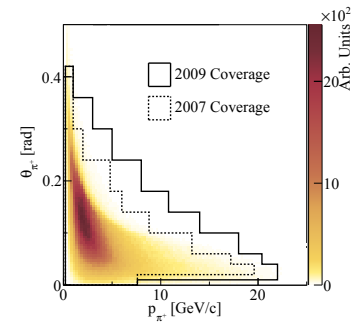

(a) $\pi^{+}$

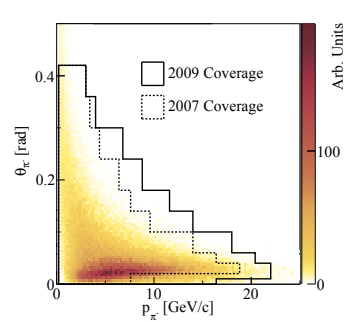

(b) $\pi^{-}$

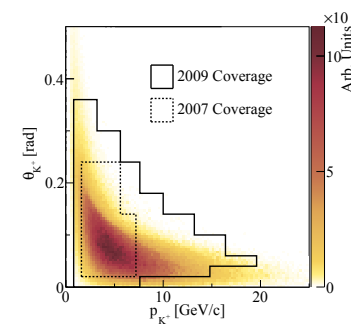

(c) $K^{+}$

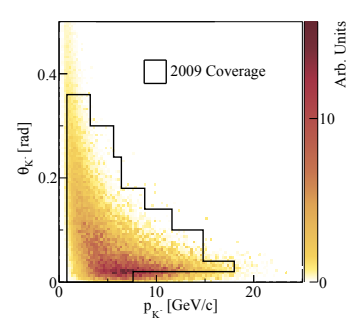

(d) $K^{-}$

Figure 1: (Colour online) The $\{p, \theta\}$ phase space of $\pi^{ \pm}, K^{ \pm}$, contributing to the predicted neutrino flux at Super-Kamiokande in the neutrino beam mode, and the regions covered by the previously published NA61/SHINE measurements [2, 3] and by the new 2009 results [12].

Particle identification in NA61/SHINE relies on measurements of the specific energy loss (dE/dx) in the TPCs and the time-of-flight measurements (tof) using the ToF-F detector. Details on spectra analysis techniques, particle identification methods as well as correction factors and uncertainties are described in great detail in Ref. [12]. Below in Figs. 2 and 3, as an example, we present the momentum distributions of $\pi^{+}\left(K^{+}\right)$and $\pi^{-}\left(K^{-}\right)$identified in $\mathrm{p}+\mathrm{C}$ interactions at $31 \mathrm{GeV} / \mathrm{c}$ for several polar angle intervals $(\theta)$.

\section{Impact of the NA61/SHINE thin target measurements on the T2K flux predictions}

Details of the T2K beam simulation and the way of the tunning of flux with NA61/SHINE results may be found in [11]. Below, we present the impact of the thin target NA61/SHINE measurements on the total T2K flux uncertainties, which are shown in Fig. 4 for $v_{\mu}$ (left) and $\bar{v}_{\mu}$ (right) enhanced beam mode. All the sources which have contributed to the flux predictions are also shown. Clearly one can see that for $v_{\mu}$ beam we can reduce the total error on the flux, in the region of energy peak around 0.6 $\mathrm{GeV}$, from $11 \%$ (obtained with 2007 NA61/SHINE data - dashed black line) below 10\% (obtained with 


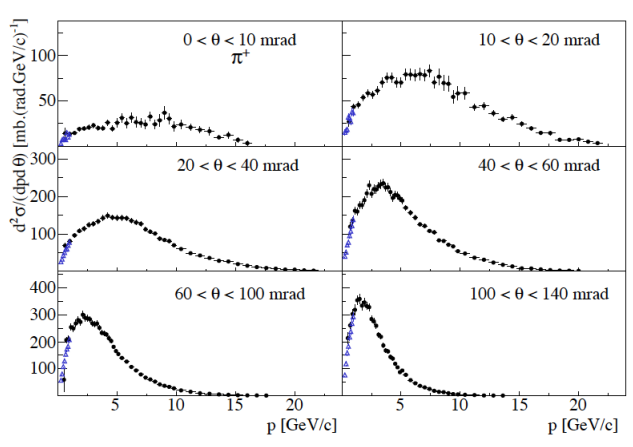

(a) $\pi^{+}$

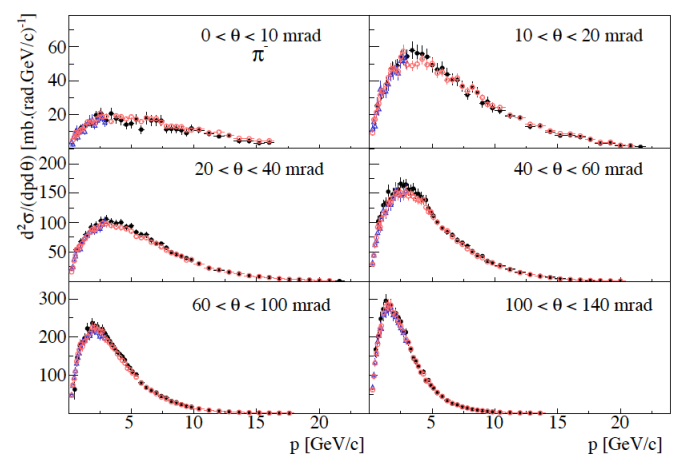

(b) $\pi^{-}$

Figure 2: (Colour online) Laboratory momentum distributions of $\pi^{+}$and $\pi^{-}$produced in $\mathrm{p}+\mathrm{C}$ interactions at $31 \mathrm{GeV} / \mathrm{c}$ production processes in selected polar angle intervals $(\theta)$. Results obtained with two (left) and three (right) different analysis techniques are presented: open blue triangles are the $d E / d x$ analysis, open red circles are the $h^{-}$analysis and full black circles are the $t o f-d E / d x$ analysis [12].

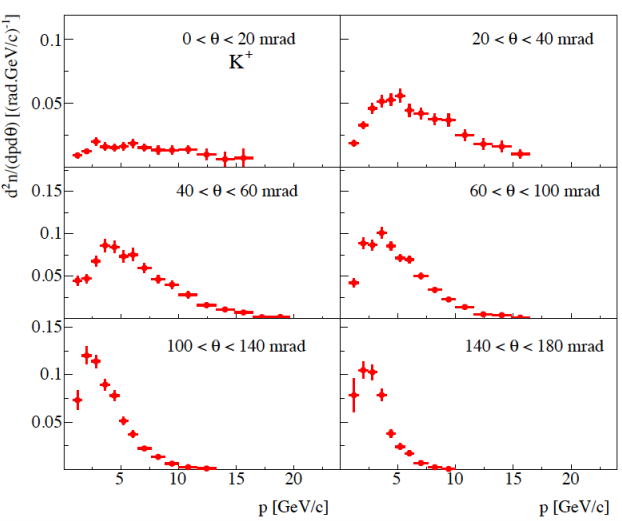

(a) $K^{+}$

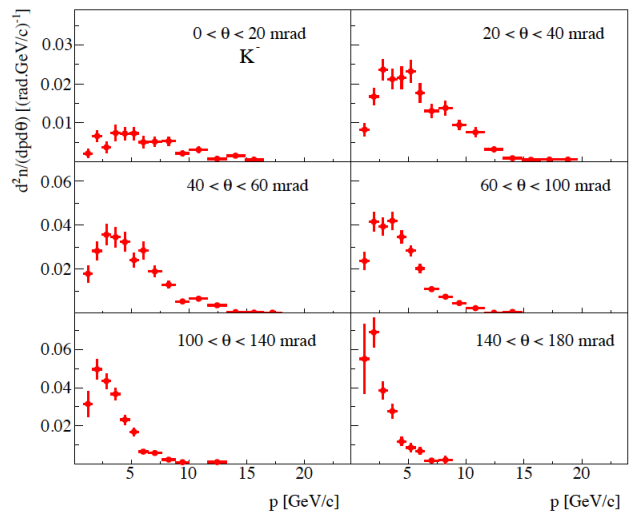

(b) $K^{-}$

Figure 3: (Colour online) Laboratory momentum distributions of $K^{+}$and $K^{-}$produced in $\mathrm{p}+\mathrm{C}$ interactions at $31 \mathrm{GeV} / \mathrm{c}$ production processes in selected polar angle intervals $(\theta)$. Results obtained with tof- $d E / d x$ analysis [12].

2009 NA61/SHINE data - solid back line). In addition the 2009 NA61/SHINE data set was for the first time used to tune $\bar{v}_{\mu}$ enhanced beam, and current status presents again below $10 \%$ total uncertainty on the flux at the energy peak. However, hadron interactions uncertainties, marked with the red lines, are still the most dominant sources in the flux predictions for both neutrino and anti-neutrino modes. We are limited with reduction of the flux uncertainties even with the dedicated measurements done on the thin target, because these measurements do not account for the re-interactions in the $90 \mathrm{~cm}$ long T2K target. The flux uncertainties can only be further improved using the NA61/SHINE measurements with the T2K replica target. 

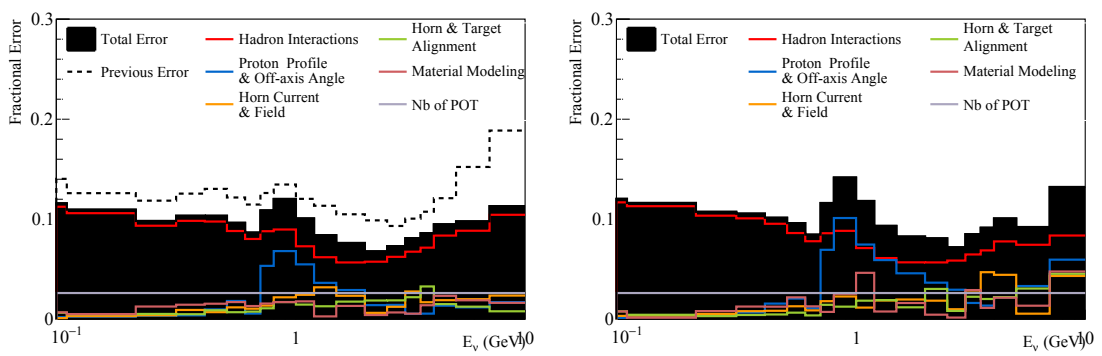

Figure 4: (Colour online) The total T2K flux uncertainties are shown for $v_{\mu}$ (left) and $\bar{v}_{\mu}$ (right) enhanced beam modes together with all the sources which contribute to the T2K flux predictions.

\subsection{The NA61/SHINE 2009 replica target results}

In addition to the published measurements on a thin target the NA61/ SHINE collaboration also performed studies of hadron emission in interactions of a $31 \mathrm{GeV} / \mathrm{c}$ proton beam with a full-size replica of the T2K target using data taken in 2007 (see Tab.1). Uncorrected differential yields of $\pi^{+}$at the surface of the replica target and their ratios with respect to the predictions of the model used to simulate hadronic interactions in the T2K target were published [13]. Moreover, there are available new measurements of fully-corrected differential yields of $\pi^{ \pm}$mesons from the surface of the T2K replica target [14]. These results were obtained using data collected during a high-statistics run performed also in 2009 (see Tab.1). Compared to the previous NA61/SHINE thin-target analyses discussed above, the $90 \mathrm{~cm}$ long T2K replica target was divided into 6 longitudinal sections, which contribute differently to the final neutrino flux (see Fig. 5). Hence, the analysis of the T2K replica target is conducted in $\{p, \theta, z\}$ bins. The longitudinal $z$ binning was determined by a study performed together with the $\mathrm{T} 2 \mathrm{~K}$ beam group. It was found that five longitudinal bins and one additional downstream bin are sufficient to obtain required neutrino flux prediction.
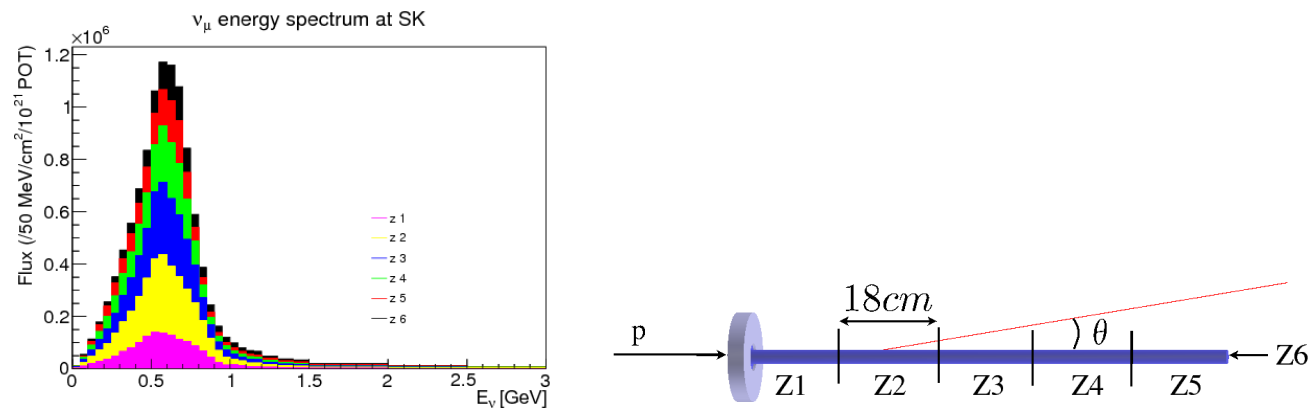

Figure 5: (Colour online) Right figure: histograms showing the contribution of each of the 6 longitudinal target bins to the muon neutrino flux at Super-Kamiokande. Left figure: a sketch of the longitudinal binning of the T2K replica target [14].

In Fig. 6 we present spectra of $\pi^{+}$at the surface of the T2K replica target, for selected polar angle intervals and for the six longitudinal bins $z$. All results which were obtained on T2K replica 
target from 2009 data set with details of the analysis, systematic error studies and more may be found in Ref. [14]. The work to use these measurements for the T2K (anti-)neutrino flux predictions in on-going.

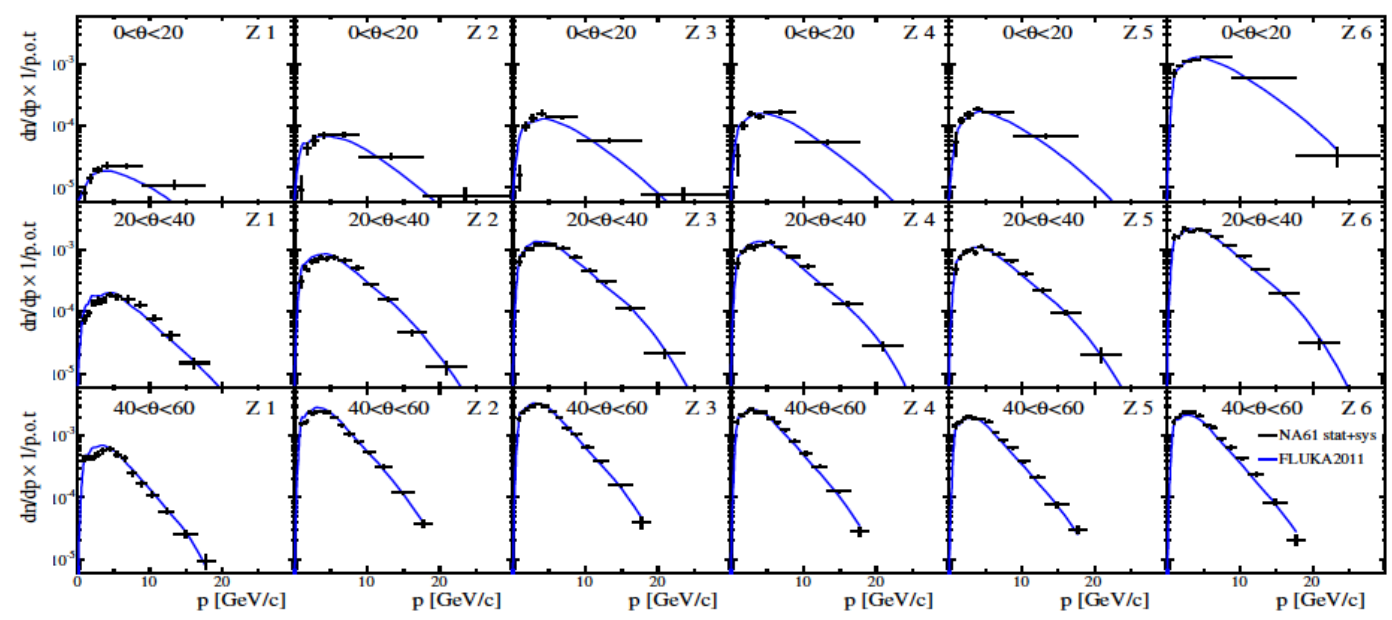

Figure 6: (Colour online) Spectra of positively charged pions at the surface of the T2K replica target, in the polar angle range from 0 to $60 \mathrm{mrad}$, and for the six longitudinal bins $z$ as a function of momentum. The FLUKA2011 prediction is overlaid [14].

\section{Acknowledgments}

Author wishes to acknowledge financial support from the National Science Centre (NCN) of Poland grant number 2014/14/M/ST2/00850.

\section{References}

[1] K. Abe et al., Nucl. Instrum. Meth. A659 (2011), 106-135

[2] N. Abgrall et al., Phys. Rev. C84 (2011) 034604

[3] N. Abgrall et al., Phys. Rev. C85 (2012) 035210

[4] N. Abgrall et al., Phys. Rev. C89 (2014), 025205

[5] N. Abgrall et al., JINST 9 (2014) P06005

[6] K. Abe et al., Phys. Rev. Lett 112 (2014), 061802

[7] K. Abe et al., Phys. Rev. D88 (2013), no. 3, 032002

[8] K. Abe et al., Phys. Rev. Lett 107 (2011), 041801

[9] K. Abe et al., Phys. Rev. Lett 112 (2014) no. 18, 181801

[10] K. Abe et al., Phys. Rev. Lett 111 (2013) no. 21, 211803

[11] K. Abe et al., Phys. Rev. D87 112 (2013) no. 1, 012001

[12] N. Abgrall et al., Eur. Phys. J. C76 (2016) no.2, 84

[13] N. Abgrall et al., Nucl. Instrum. Meth. A701 (2013) 99-114

[14] N. Abgrall et al., arXiv:1603.06774 to appear in Eur. Phys. J. C (2016) 